\title{
Acercar a los hoteles con sus clientes mediante las TIC
}

\author{
Sandra Dinora Orantes, Graciela Vázquez Álvarez \\ y Ricardo Tejeida Padilla
}

\begin{abstract}
Resumen
Esta investigación analiza la medida en que los hoteles mexicanos y particularmente las pequeñas y medianas empresas (PYMES) han desarrollado una estrategia que les ayuda a gestionar las relaciones con los clientes. En este artículo se intenta identificar las prácticas que aparecen con frecuencia en el proceso de comercialización de una empresa, para lograr una adecuada relación con sus clientes por medios tradicionales o a través de internet, observando las acciones básicas del manejo de la información del personal y de suministros de productos y servicios. También se consideran los efectos de una gestión de relaciones con los clientes en su lealtad y satisfacción, así como el impacto de las prácticas de retención y adquisición de nuevos mercados y compradores. Por otra parte, se busca lograr el establecimiento de las bases de una estrategia relacional, para apoyarse posteriormente en una herramienta electrónica, que ayudará al negocio hotelero para gestionar eficazmente servicios, productos y reservas, y así proporcionar a sus clientes un servicio lo más rápido y eficaz posible.
\end{abstract}

Palabras clave: gestión de las relaciones con los clientes, PYMEs hoteleras, satisfacción, lealtad, estrategias relacionales de negocio.

\section{BRINGING HOTELS CLOSER TO THEIR CUSTOMERS THROUGH ICT}

\begin{abstract}
This research analyzes the extent to which Mexican hotels and particularly Small and Medium-sized Enterprises (SMES) have developed a strategy that helps them to manage customer relations. In this article we try to identify the practices that frequently appear in the commercialization process of a company in order to achieve an adequate relationship with clients by traditional means or through the Internet, observing the basic actions of information management of personnel and supplies of products and services. The effects of a customer relationship management are also considered in terms of loyalty and satisfaction, as well as the impact of retention and acquisition practices of new markets and buyers. On the other hand, we seek to establish the basis of a relational strategy, to subsequently rely on an electronic tool that will help hotel businesses to effectively manage services, products and reservations, and thus to provide its customers with a service as fast and efficient as possible.
\end{abstract}

DOI: http://doi.org/10.22201/codeic.16076079e.2019.v20n3.a2 
Keywords: customer relationship management, SMES in the hotel industry, satisfaction, loyalty, business strategies.

\section{Sandra Dinora Orantes Jiménez}

Docente investigadora del Centro de Investigación en Computación (CIC), Departamento de Investigación en Computación, Laboratorio de bases de datos y tecnología de software, Instituto Politécnico Nacional (IPN).

\section{Graciela Vázquez Álvarez}

Doctora en Ciencias de la Computación por el CIC del IPN, maestra en Ciencias con especialidad en Sistemas de Información e Ingeniero Industrial con diplomado en Habilidades Docentes por el Instituto Tecnológico de Estudios Superiores de Monterrey, campus Estado de México. Diplomado en Docencia para profesores de Enseñanza Superior en la Dirección de Estudios Profesionales del IPN. Diplomado en el Desarrollo del Potencial Creativo en la Escuela Superior de Comercio y Administración, IPN. Diplomado en Diseño y Programación Orientada a Objetos en Plataforma J2EE, en la Dirección de Cómputo y Comunicaciones del IPN. Diplomado en Coaching aplicando la Programación Neurolingüística, en el CECYT Miguel Othón de Mendizábal del IPN. Diplomado en Ciencia y Tecnología, en la ESIME-Zacatenco del IPN. Diplomado en Desarrollo Humano Integral en la ESCA-Sto. Tomas del IPN. Profesor Investigador de la Sección de Estudios de Posgrado e Investigación de la ESIME-Zacatenco desde el 2001 a la fecha; materias impartidas en el programa de Posgrado en Ingeniería de Sistemas, en la línea de Sistemas de Información y Bases de Datos.

\section{Ricardo Tejeida Padilla}

ricardotp75@gmail.com

Es Ingeniero Industrial, tiene el grado de maestro en Ciencias en Ingeniería de Sistemas y de doctor en Ciencias con especialidad en Ciencias Administrativas. Ha publicado más de 20 artículos en revistas indexadas y siete capítulos en libros, cinco de ellos en inglés en editoriales de prestigio internacional. Ha dirigido más de cinco proyectos de investigación, cuatro tesis de doctorado, 27 tesis de maestría y una tesis de licenciatura. Es miembro del Sistema Nacional de Investigadores (SNI) nivel I. Su adscripción actual es en la Escuela Superior de Turismo (EST) del IPN. Su línea de investigación es la Sistémica. En 2009 ganó el premio de la mejor tesis de posgrado como director. En 2005, ganó el Premio a la mejor tesis de posgrado con su tesis doctoral y recibió la Presea Lázaro Cárdenas en nivel doctorado. Es miembro activo en asociaciones de prestigio internacional como la International Society for the Systems Sciences (ISSS), la Asociación Latinoamericana de Sistémica (ALAS), y la Academia Mexicana de Ciencia de Sistemas (AMCS). 


\section{Introducción}

En la actualidad se ha introducido la tecnología de internet a los negocios internacionales, lo cual ha llevado a su aplicación generalizada en la industria hotelera (Law y Hsu, 2006). De esta manera, el mercadeo de los hoteles a través de internet cada vez se hace más necesario y ayuda a las empresas a establecer relaciones interactivas con sus clientes y atender sus necesidades y preferencias (Zineldin, 2000), de una manera personalizada. A medida que crece la competencia, el cliente es más exigente y el negocio del hospedaje necesita desarrollar nuevos productos y prácticas orientadas a sobrevivir y retener a sus clientes; la satisfacción del cliente constituye un factor significativo que crea una fuerte ventaja competitiva y ofrece motivos decisivos para un mayor avance de las empresas. Según Zineldin (2000) "los hoteles tienen la posibilidad de recibir retroalimentación de los clientes", la cual puede ir desde clientes realmente satisfechos hasta otros con quejas. En este contexto, es pertinente para las pequeñas y medianas empresas (PYMES) implementar estrategias de gestión de las relaciones con los clientes (CRM, del inglés Customer Relationship Management), como parte de un proceso natural. Por lo tanto, es importante enfatizar que la gestión de las relaciones con los clientes es una estrategia de negocios, no solamente un software que las empresas deben adquirir.

Estudios experimentales muestran que, a partir del establecimiento de una estrategia CRM, la relación de mercadotecnia puede tener un impacto positivo en la satisfacción del cliente y, a la vez, ayuda a identificar posibles consumidores. Desde una perspectiva de mercadeo relacional ${ }^{1}$ puede mencionarse que la industria hotelera debe emplear todo el potencial de las estrategias de negocios como una herramienta para facilitar el desarrollo de las relaciones con los clientes y, además, apoyarse en las tecnologías de la información (TI) (Berry y Parasuraman, 1991).

Este artículo busca apoyar a aquellos quienes toman decisiones estratégicas en las organizaciones hoteleras, por lo tanto, se muestra cómo la gestión de relaciones con el cliente cambia radicalmente la posición estratégica de las empresas que lo implementan. Si bien se tienen que reconsiderar las prácticas de negocio de la PYME respecto a sus clientes, es importante entender

1 ¿Qué es el mercadeo relacional? "el proceso social y directivo de establecer y cultivar relaciones con los clientes, creando vínculos con beneficios para cada una de las partes, incluyendo a vendedores, prescriptores, distribuidores y cada uno de los interlocutores fundamentales para el mantenimiento y explotación de la relación" (Universidad del Rosario).

\section{La importancia de la satisfacción del cliente}

Cada vez que un servicio o producto no satisface inmediatamente queda grabado en la mente de la gente todo ser humano recuerda más fácilmente aquello que no satisface que lo que sí lo ha hecho. Pero ¿cuál es la razón de esto?

Podrían pensarse varias explicaciones. En primer lugar, cuando se compra un producto o servicio se tienen ciertas expectativas y se espera obtener aquello por lo que se paga. También está claro que con la actual oferta y demanda cada 
vez se espera más. Actualmente, hay productos y servicios con los que antes no se podía contar, productos con los que las generaciones previas ni siquiera soñaron y ha sido fácil habituarse a ellos. Esto lleva a que las personas sean cada vez más exigentes, debido a que también la vida se ha vuelto más complicada, con mayores niveles de estrés.

Por lo tanto, cada vez es más difícil agradar a los clientes y lograr satisfacerlos. Incluso si los clientes están completamente satisfechos con un producto o servicio, nada impide que un grupo de ellos empiece a hacer negocios con la competencia.

La relación con el cliente tiene un ciclo de vida según Stauss, Gouthier y Seide (2007), el desarrollo de una relación de negocios se expresa inicialmente por el aumento de la intensidad de la relación, que más adelante caerá en declive. Si bien es importante el nivel de ingresos de los clientes, la intensidad de la relación es mucho más importante.

Si partimos de que la satisfacción del cliente es la principal norma para la teoría y la práctica del marketing y la gestión, es posible emplear series de tiempo que permitan dar seguimiento a los clientes, pues las empresas deben centrarse en su bienestar. Por lo tanto, es importante generar reportes de gestión (Balvers et al., 2015). En este sentido, debe buscarse la forma de tener indicativos que nos muestren si lo que se está haciendo en la empresa, en tanto a la satisfacción y lealtad del cliente, va bien o dónde va mal. En cualquier empresa la retroalimentación por parte del cliente es valiosa y una de las técnicas que se emplean para medir y dar seguimiento es el empleo de encuestas, aunque debe aclararse que no es la única.

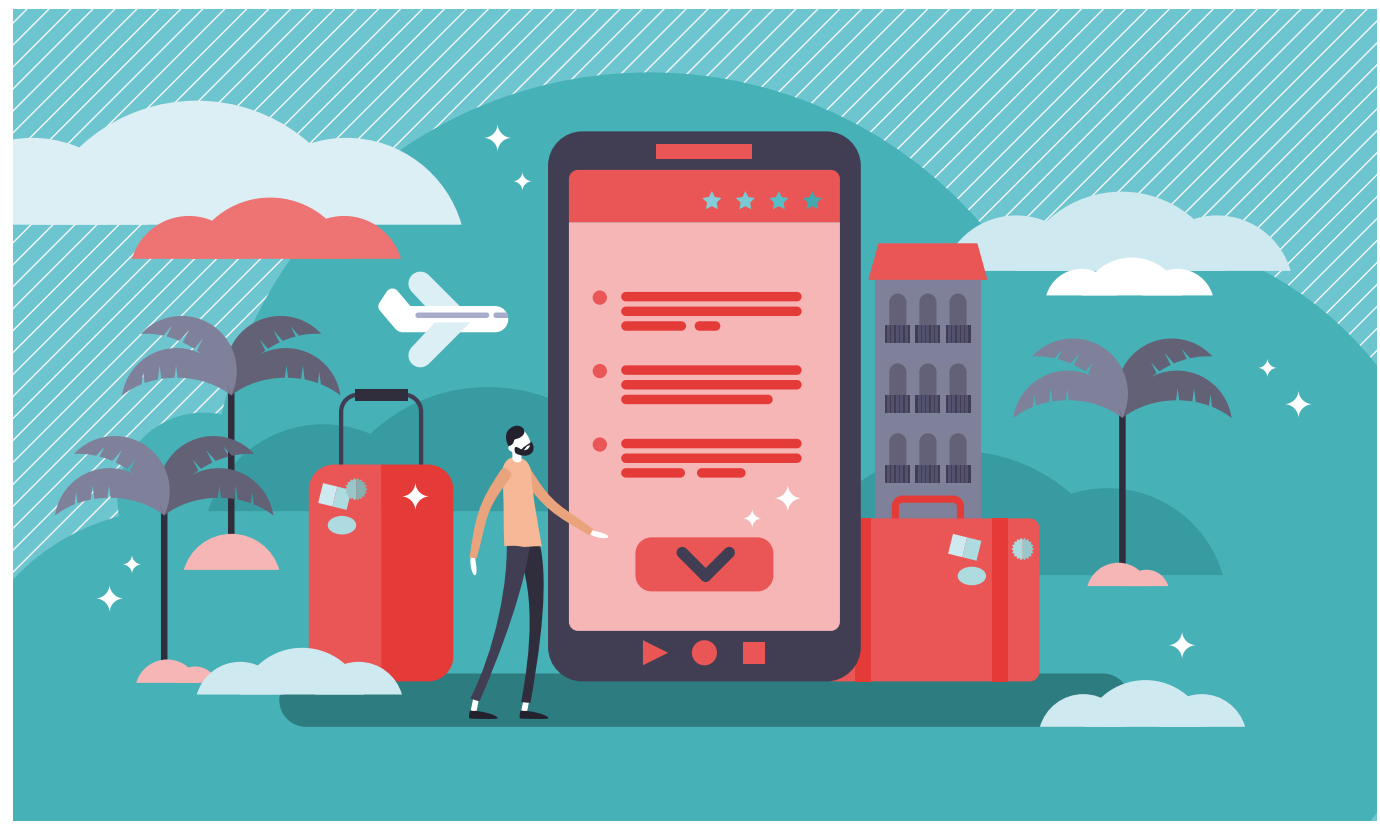


Las PYMES, que fácilmente pueden ser devoradas por las empresas grandes y también por la competencia de su misma categoría, deben poner especial atención en tomar como base estrategias de negocio que las ayuden a seguir en el mercado, como la gestión CRM.

Cualquier medio que se emplee para considerar al cliente, sus opiniones y necesidades respecto a un producto o servicio, es sólo la primera parte; lo principal es la evolución de la empresa, que debe incluir la participación y formación del personal. Lo importante de la aplicación de una estrategia de negocio relacional es el resultado, que es económicamente beneficioso, debido a una menor pérdida de clientes, mayor posicionamiento en el mercado, productos y servicios más fuertes, logrando así una mejor reputación de la empresa.

Existen investigaciones como la de Lin y colaboradores (2016) que se enfocan en determinar la relación entre la satisfacción del cliente y empleados satisfechos dentro de la empresa; estos autores mencionan que la creación de satisfacción del cliente ha sido el objetivo principal de las empresas de servicios. Sin embargo, también se ha estudiado cómo repercute la satisfacción de los empleados en la mejora de la satisfacción del cliente; sin embargo, no hay muchos estudios que exploren este mecanismo de vinculación, pero se considera que es un factor importante que debe tomarse en cuenta.

Por otra parte, Habel y Klarmann (2015) resaltan aún más la importancia del desenvolvimiento de los empleados en una estrategia orientada al cliente; ha estudiado cómo perder buenos trabajadores puede ocasionar insatisfacción en los consumidores. Ellos mencionan que su estudio tiene importantes implicaciones para los gerentes, quienes deben ser conscientes de que en función de su categoría de empresa y del producto o servicio la reducción de los empleados tiene efectos en los clientes. Los gerentes deben considerar alternativas a la reducción de personal, sobre todo si los clientes no consideran la marca un importante criterio de compra, sino que toman en cuenta la forma en que son atendidos por determinados empleados de la organización.

Las implicaciones de aplicar una estrategia de negocios orientada al cliente

${ }^{2}$ Convocatoria a solicitud de la Dirección General de Mejora Regulatoria, a través del Fondo Sectorial del

Consejo Nacional de Ciencia

y Tecnología (CONACYT) y

la Secretaría de Turismo

(SECTUR) para la Investigación, el Desarrollo y la Innovación Tecnológica en Turismo, la SECTUR y el Centro de Estudios Superiores en Turismo (CESTUR) -ya sean encuestas de satisfacción o cualquier otro método que se empleevan más allá y sólo tendrán éxito si cuentan con el pleno apoyo de la dirección y si se involucra a toda la organización en su implantación.

\section{La gestión de relaciones con los clientes para el sector hotelero}

La gestión de las relaciones con los clientes (CRM) se utiliza para definir una estrategia de negocio enfocada a los clientes, en la que el objetivo es reunir la mayor cantidad posible de información sobre ellos para generar relaciones a largo plazo y aumentar así su grado de satisfacción (Membrado, 2002). 
Cada cliente es diferente, por tanto, requiere productos y servicios de acuerdo a su personalidad o necesidades (negocios o turismo). Para el sector hotelero la competencia es grande y quienes se posicionan mejor son las cadenas hoteleras con hospedajes de cuatro estrellas en adelante, que fueron las primeras en aplicar estrategias como CRM.

Los hoteles, para intentar mantener la lealtad del cliente, buscan su satisfacción y crean programas como clientes oro, plata y bronce, donde de acuerdo con la categoría, depende el servicio. Cada categoría etiqueta al cliente y lo importante es que éste se sienta cómodo con ella. Lo que toda empresa hotelera no debe perder de vista es que cada cliente necesita y quiere diferentes cosas y que lo que requieren ante todo es ser tratados como individuos, como personas.

En 2011 se publicó una convocatoria para desarrollar un nuevo Sistema de Clasificación Hotelera Mexicano $(\mathrm{SCH})^{2}$ y dotar así al sector de una herramienta de medición sólida que permita no sólo indicar la cantidad de alojamiento disponible sino también las cualidades del mismo (Instituto de Competitividad Turística [ICTUR], 2014). Con base en estos clasificadores, el sector hotelero puede establecer una estrategia adecuada de gestión de las relaciones con los clientes.

De esta manera, si una PYME del sector hotelero decide adoptar una estrategia cRm no le implica altos costos. Con las ventajas del cómputo en la nube, particularmente en el modo Software as a Service (SaaS), ya existen aplicaciones donde sólo basta tener una conexión a internet para hacer uso del llamado Cloud CRM, lo que lleva a afirmar que las Pymes también pueden competir empleando las ventajas de las tecnologías de información (TI).

Cuando se trata de hoteles, el número de estrellas refleja de modo intuitivo el tipo de oferta (ver figura 1). Hoteles con cualidades superiores obtienen mayor número de estrellas al ser evaluados (ICTUR, 2014). Existe también la calificación con medias estrellas, lo cual permite incrementar la precisión en la evaluación: da flexibilidad a la herramienta y limita la sobreestimación en la autoclasificación, pues al utilizar exclusivamente estrellas completas, en ocasiones, se permiten diferencias importantes entre los hoteles de una misma categoría y se generan pocos incentivos en los hoteleros para mejorar sus establecimientos, ya que los requerimientos de inversión para ascender una estrella completa podrían ser muy elevados con respecto al capital disponible o a los beneficios económicos esperados por la mejora en sus instalaciones (ICTUR, 2014).

Figura 1. Significado del número de estrellas con que se califica (ICTUR, 2014).
Una estrella: solo ofrece lo indispensable.

Dos estrellas: servicios e infraestructura básicos

Tres estrellas: instalaciones adecuadas, servicio completo y estandarizado, sin grandes lujos.

Cuatro estrellas: instalaciones de lujo y servicio superior.

Cinco estrellas: instalaciones y servicios excepcionales 
La ventaja de que la Secretaría de Turismo (SECTUR), a través del ICTUR, establezca esta clasificación es que permite a las empresas del sector hotelero saber exactamente qué ofrecer a los clientes, hasta dónde quieren competir y qué escala quieren alcanzar.

Los hoteleros ahora saben hasta dónde quieren llegar y cuentan con una base para que los clientes estén satisfechos, mantengan su lealtad y continúen visitando determinados establecimientos e incluso recomendándolos.

Los clientes ahora pueden relatar, ya sea a través de encuestas o por el medio que elija el sector hotelero, la experiencia de uso en determinados establecimientos de hospedaje, tomando en cuenta variables relacionadas al ambiente físico del hotel, al personal de servicio, al producto de alojamiento adquirido, a la interacción con otros huéspedes (ambiente del hotel) y también elementos de comunicación presentes. La experiencia turística es

\section{Adoptar una estrategia} de CRM es parte de la naturaleza del negocio y en un futuro se deberá hacer uso de las TIC, ya que es la única manera de llegar a otros posibles mercados y diversa y los huéspedes buscan diferentes espacios para alojarse, según la ocasión y el motivo del viaje.

Por tanto, este sistema adecua el instrumento y las variables valoradas para asegurar que cada tipo de hotel sea categorizado con base en los atributos que son más trascendentes para los huéspedes a los que están enfocados su propuesta y su producto. Por ejemplo, para el huésped de un hotel de negocios no debiera ser prioritario que el establecimiento cuente con instalaciones deportivas; en el caso de un hotel boutique el huésped no esperaría grandes salones de reuniones e instalaciones para banquetes, ni el huésped de un hotel ecológico, construcciones ostentosas ni cualquier otro servicio que generara un impacto ambiental negativo (SECTUR, 2014).

Esta investigación se centra en la categoría cama y desayuno (cyD), dentro de las Pymes hoteleras; este tipo de establecimiento está dirigido a turistas que generalmente no buscan permanecer en el hotel a lo largo del día, pues su prioridad es visitar la ciudad, el pueblo o la región donde está asentado el establecimiento. Por esta razón, su ubicación es importante, ya sea céntrica o próxima a espacios conectivos o vías de transporte. En México, los cy están dirigidos primordialmente a amantes del turismo cultural, que buscan un lugar donde dormir y desayunar, así como que sea óptimo para salir a recorrer los sitios aledaños al inmueble (SECTUR, 2014).

También podría considerarse la categoría de los llamados hoteles express, donde los huéspedes son personas cuyo motivo principal de viaje es empresarial y de negocios. Estos turistas acuden a trabajar a lugares fuera de su residencia habitual y necesitan un espacio apropiado a su rol laboral para pasar 
la noche. Estos establecimientos cuentan sólo con servicio de desayunos, siendo éste el único alimento completo que ofrecen. Es común que proporcionen transportación gratuita a las terminales y centros de negocios. Podría parecer que satisfacer a clientes en este tipo de hotel resulta una tarea poco complicada, pero no es así. Si se desea cautivar su atención, las instalaciones deberán ser acogedoras, brindar un desayuno variado y agradable y, como ya se mencionó, tener una excelente ubicación y atención por parte de los empleados, los cuales definitivamente deberán hablar más de un idioma, por el tipo de clientela que los visitará.

Todos estos puntos van conformando el CRM, y es importante determinar un buen método de evaluación de la satisfacción del cliente. El cuestionario-encuesta breve sigue ocupando primer lugar, pero no debe ser conducente, ya que realmente lo que interesa saber es lo que el usuario piensa y siente sobre su estadía.

La base del CRM para una empresa hotelera es mantener el siguiente pensamiento: se obtendrá más satisfacción de los clientes y su lealtad cuando se tome el tiempo de estar al pendiente de ellos y tratarlos con la importancia que se merecen.

\section{Barreras en la adopción de CRM}

Jiménez-Zarco y colaboradores (2007) mencionan que:

[...] abundantes estudios han tratado de determinar las causas que han motivado el desarrollo de la economía del conocimiento (Knowledge Economy), que es el sector de la economía que utiliza el conocimiento como elemento fundamental para generar valor y riqueza por medio de su transformación a información. Pero entre todos los factores identificados la literatura especializada reconoce en la globalización, el cambio en los patrones de comportamiento de los individuos y en el uso intensivo de las Tecnologías de la Información y de las Comunicaciones (TIC), a los verdaderos responsables de la aparición de la nueva economía. De forma efectiva, estos fenómenos han afectado en gran medida a las empresas, aunque la forma de ser entendidos y el impacto que cada uno de ellos genera sobre las organizaciones viene determinado por el tipo de empresa y por el entorno donde se ubica.

Las posibles barreras para que las PYMEs del sector hotelero adopten tecnologías y estrategias enfocadas en los clientes, como CRM, son problemas relacionados con el tamaño de los establecimientos y la falta de filiación a una cadena (Arvanitis y Stern, 2001; Schegg et al., 2007). Esto hace más difícil de convencer a las pequeñas empresas hoteleras de que, sin importar que sus recursos sean menores, una estrategia cRm que los beneficie puede ser establecida.

Las grandes cadenas hoteleras tienen la capacidad de adquirir herramientas para adoptar un sistema Enterprise Resource Planning (ERP), y utilizan herramientas de software con toda la gama de funciones, donde cada función se puede dividir en módulos. Planifican todos los recursos de la empresa hotelera y saben exactamente cuándo y dónde actuar. Una de las herramientas en las que 
han invertido es Oracle E-Business Suite o bien en un programa para aplicaciones de negocios alemán conocido como SAP. Ambas herramientas de software no son gratuitas, requieren licencia de uso y no son baratas, por lo que no están dirigidas a empresas pequeñas. Son programas empleados por grandes organizaciones.

Pero para el caso de las Pymes, que no necesitan disponer de una gama completa de funciones, es posible emplear sólo ciertos módulos: lo que más las apoya es la estrategia CRM. En muchos casos, las PYMEs compiten simultáneamente con las grandes empresas por el mismo cliente. Por eso necesitan soluciones que sean fáciles de implementar y mantener, y que exijan pocos recursos de su equipo de tecnologías de información.

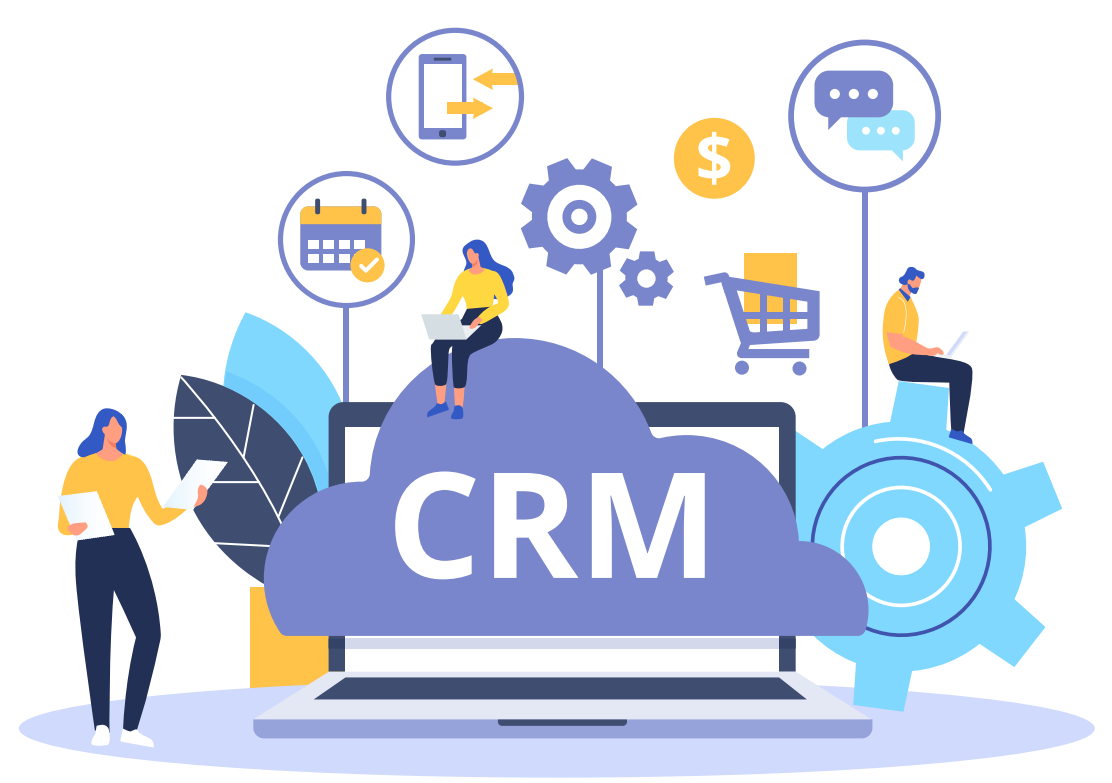

No obstante, las desigualdades en el nivel de adopción de CRM presentadas por el establecimiento de diversas condiciones no parecen estar relacionadas sólo al tamañoy a la filiación; aunque, ciertamente, tamaño y filiación junto con otras características, como el tipo de servicio que se ofrece, significan una forma diferente de implantar la estrategia CRM. Así, las soluciones deben dirigirse a las empresas hoteleras, prestando atención a sus diferentes características y requerimientos. Con esto se garantizará que las Pymes del sector hotelero entiendan la necesidad de invertir en estrategias de negocio como CRM y, posteriormente, en TIC de acuerdo a sus exigencias.

Adoptar una estrategia de cRM es parte de la naturaleza del negocio y en un futuro se deberá hacer uso de las Tic, ya que es la única manera de llegar a otros posibles mercados y clientes como China, Japón y otras partes del mundo, que de otra forma no les serían accesibles. Se trata, en principio, de la aceptación y comprensión de la estrategia CRM, y, luego, de la inversión en tecnología.

Existen herramientas TIC determinantes para la admisión de la estrategia CRM en las PYMEs hoteleras; por ejemplo, la teoría de la acción razonada (TRA, del inglés Theory of Reasoned Action) y el modelo de aceptación tecnológica (TAM, del inglés Technological Acceptance Model), por mencionar algunas.

Para lograr una adecuada gestión del conocimiento y que éste sea una fuente de creación de valor para la empresa, el primer paso es identificar el conocimiento que se genera en su interior, así como aquel que proviene del exterior, para después sistematizarlo y compartirlo entre todos los miembros de la organización (Demuner Flores et al., 2015). 
Lo importante es que la PYME hotelera completa, pasando por toda la jerarquía de la organización, tome el CRM, lo convierta en parte de su filosofía y que hasta el último miembro del establecimiento acepte esta estrategia para bien de toda la empresa.

\section{Casos de éxito}

Actualmente, la industria en general se enfoca en hablar de que cRm falla; muchas empresas, por temas de confidencialidad, no hablan de sus casos de éxito. Lo mismo sucede con el sector hotelero que, para no dar a conocer sus debilidades, no muestra en qué fue posible mejorar con la aplicación de un CRM.

A continuación se describirán los pocos ejemplos que pueden mencionarse dentro del sector hotelero en México, en donde la implantación de una estrategia de negocios como cRM ha sido exitosa. Es importante aclarar que solamente el segundo caso se clasifica como PYME, los otros dos son cadenas de hoteles a nivel internacional.

- Grupo Sunset World (2015). Es una cadena mexicana de hoteles de lujo localizada en Cancún y la Riviera Maya. Emplea a 1500 personas y se enfoca principalmente al hospedaje tradicional y al tiempo compartido. Edgar Osorio, director de sistemas de información del Grupo (cıo por sus siglas en inglés), habló en 2011 sobre la manera en que obtuvieron visibilidad, redujeron costos y mejoraron la toma de decisiones tras la implementación de la suite Business Intelligence del proveedor sueco QlikTech. Detalla quiénes y qué departamentos han sido los principales beneficiados con el uso de la herramienta y los requisitos técnicos de implementación. Menciona que una de las áreas explotadas es la obtención y retención de clientes mediante su satisfacción (Cota, 2009).

- Un hotel cerca de la Expo Guadalajara, cuya oferta es limitada por el número de habitaciones, en este caso 70, plantea el aumento de la rentabilidad usando Zoho cRM y fidelizando así a los clientes más rentables. Por lo tanto, la primera tarea fue definir "qué es un cliente rentable" y luego identificarlos (Arce, 2011).

- RCD Resorts. Más conocido por su marca Palace Resorts, ha emprendido un cambio evolutivo para convertirse en Hard Rock Hoteles, una cadena de hoteles de nivel internacional, que ofrece al turista todas las comodidades y servicios de un gran hotel en sus paradisiacos destinos. Hoy en día Hard Rock Hoteles tiene presencia en Estados Unidos, México y República Dominicana, en las localidades de Cancún, Riviera Maya, Playa del Carmen, Cozumel, Puerto Vallarta y Punta Cana. Cuenta con 11 resorts, que están en proceso de adaptarse a la imagen de Hard Rock Hoteles. Jaime García, Director de TI de RCD Resorts, comenta lo importante que es contar con un sistema como SAP: "SAP es vital como soporte de nuestras operaciones y plan de expansión. Específicamente con SAP ECC y SAP CRM estamos apuntalando el core central de nuestra gestión" (NOVIS, 2012). 


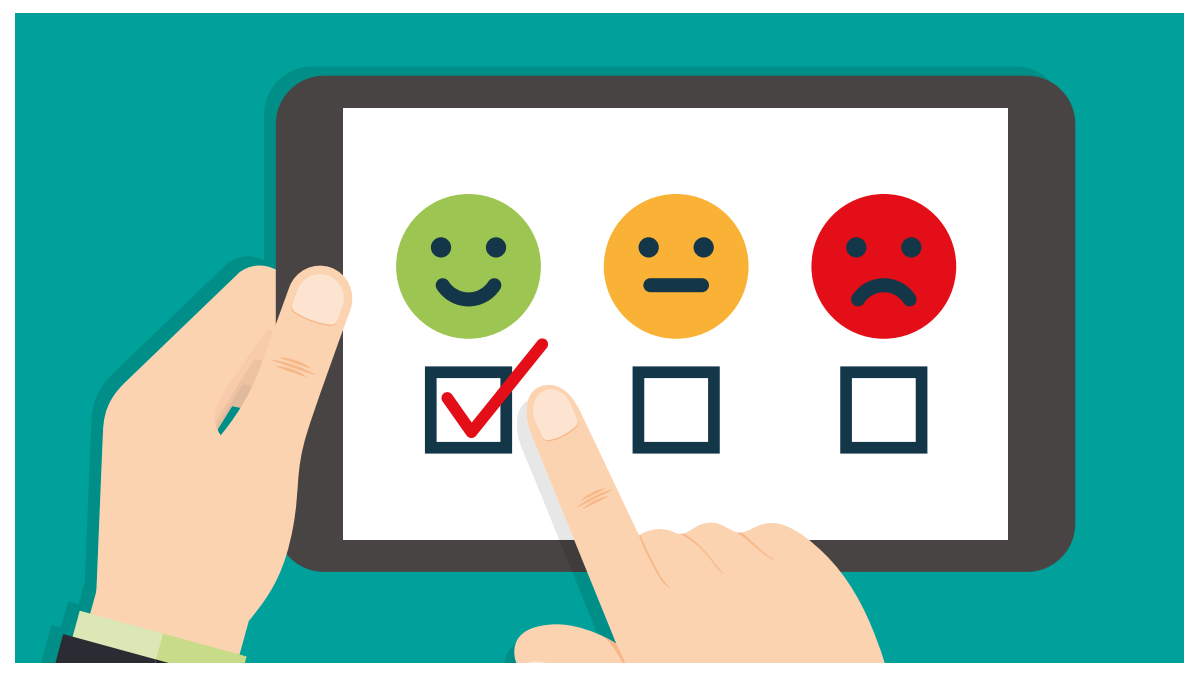

\section{Discusión}

Las PYMES de cualquier sector necesitan una guía que los apoye en la elección de una tecnología (Orantes et al., 2015). Por otra parte, se hace necesario crear conciencia del patrimonio del país en las personas jóvenes, pues son los principales promotores del turismo, quienes en principio pueden atraer a otros jóvenes a visitar México, para apoyar al turismo y a un sector importante como es el hospedaje. Es preocupante que "Ios resultados de una evaluación diagnóstica indican que los estudiantes tienen pocos referentes sobre los bienes culturales y naturales del país y en consecuencia, muestran evidencias de una escasa conciencia de su valor patrimonial, así como dificultades para proponer distintos aprovechamientos" (Rico Marsard y Alucema, 2010).

\section{Conclusiones}

Las PYMES del sector hotelero deben estar dispuestas a ofrecer servicios personalizados a las necesidades de cada cliente. Asimismo, la estrategia CRM debe estar presente tanto para mantener como para adquirir nuevos clientes.

Es necesario emplear canales como internet, además de los medios tradicionales, para implementar estrategias de mercadeo relacional. Lo importante es que los procesos CRM deben estar bien diseñados y deben tenerse claras las perspectivas de los clientes en la construcción de relaciones con el establecimiento hotelero.

Se debe aspirar a que las personas que dirigen la PymE hotelera sean conscientes de los diferentes efectos de cada etapa de cRm y que, por consiguiente, planifiquen estrategias apropiadas para conocer a los clientes, generar y reforzar la satisfacción y el compromiso. 
Debe ponerse énfasis en los empleados de la organización para asegurarse de que hay comunicación entre ellos en las diferentes áreas del hotel. Deben aplicar eficientemente las estrategias de mercadeo relacional, enfatizando en la retención de clientes y la satisfacción continua.

Todos deben estar conscientes de las mejoras que conlleva la adopción de CRM. Hay que tener en cuenta que el conocimiento sobre los clientes es esencial para que el negocio crezca y para saber dónde se puede mejorar y lo que se debe ampliar y personalizar a partir de información adecuada. El objetivo es lograr no sólo satisfacción, sino una mayor lealtad impulsada por los servicios que el hotel brinda.

Para ofrecer servicios de alta calidad no importa que se trate de una PYME, hay que centrarse en las preferencias del cliente con el fin de mantenerlo y adquirir otros nuevos. Al personalizar la experiencia, se establecen los medios para una mejor comunicación con el cliente y se logra, con ello, su retención. De esta manera, la pYME hotelera obtendrá valiosos beneficios a largo plazo.

En la actualidad ya es posible que una PYME implemente una estrategia CRM y un software que la apoye, solamente tendrá que buscar soluciones económicas, que a su vez sean de calidad. Es importante, además, que sean soluciones que acompañan el crecimiento del negocio. Otras características para el uso de herramientas es que sean soluciones rentables, listas para ser empleadas, específicas para el sector y mercado, y saber que los proveedores del servicio son colaboradores en los que pueden confiar.

Lo que debe dejarse claro, es que CRM es más que una herramienta de software para ventas. CRM es una estrategia de negocio que procura la satisfacción del cliente para lograr su retención y fidelidad.

\section{Referencias}

* Arce, M. A. (2011). Incremento de la Rentabilidad de un Hotel utilizando CRM. Revista Marcas y Turismo. Recuperado de: http://www.turismoytecnologia.com/ component/k2/item/981-como-aumentar-la-rentabilidad-de-un-hotel-utilizandocrm.

Arvanitis, S. y Stern, S. (2001). The determinants of the adoption of advanced manufacturing technology. Economics of Innovation and New Technology, 10, 377414.

* Balvers, R. J., Gaski, J. F. y Mcdonald, B. (2015). Financial Disclosure and Customer Satisfaction: Do Companies Talking the Talk Actually Walk the Walk? Journal of Bussiness Ethics, 139 (1), 29-45. Dol: https://doi.org/10.1007/s10551-015-2612-6. 
* Berry, L. L., Parasuraman, A. (1991). Marketing Services. Competing hrough Quality. Lexington: Free Press/Lexington Books.

* Cota, M. (2009). Entrevista a Edgar Osorio, Business Intelligence en una cadena de hoteles de lujo mexicana. El clo de Sunset World habla de su experiencia con QlikView. Recuperado de: http://www.bi-spain.com/articulo/69311/ business-intelligence/turismo/business-intelligence-en-una-cadena-de-hotelesde-lujo-mexicana-el-cio-de-sunset-world-habla-de-su-experiencia-conglikview\#sthash.Prpkc7ya.dpuf

* Demuner Flores, M. R., Nava Rogel, R. M. y Mercado Salgado, P. (2015). Las TI como facilitadoras de la gestión del conocimiento empresarial. Revista Digital Universitaria, 16(2) Recuperado de: http://www.revista.unam.mx/vol.16/num2/ art15/index.html.

* Habel, J. y Klarmann, M. (2015). Customer reactions to downsizing: when and how is satisfaction affected? Journal of the Academy of Marketing Science, 43(6), 768789. Dol: https://doi.org/10.1007/s11747-014-0400-y.

* Instituto de Competitividad Turística (ICTUR). (2014). Sistema de Clasificación Hotelera Mexicano. Recuperado de: http://sch.sectur.gob.mx:8080/SCHWEB/.

* Jiménez-Zarco, A. I. y Martínez Ruiz, M. P. (2007). El impacto de las TIC en los procesos de innovación empresarial. Revista Digital Universitaria, 8(3). Recuperado de: http://www.revista.unam.mx/vol.8/num3/art20/int20.htm.

- Law, R.yHsu,C.H.C.(2006). Importance ofHotelWebsite Dimensions and Attributes: Perceptions of Online Browsers and Online Purchasers. Journal of Hospitality \& Tourism Research, 30(3), 295-312. Dol: https://doi.org/0.1177/1096348006287161.

Lin, J. S. C., Liang, H. Y. y Chu, C. Y. (2016). Satisfying Customers through Satisfied Employees: Exploring the Emotional Mechanism Linking Employee Satisfaction and Customer Satisfaction. En Petruzzellis L., Winer R. (Eds.), Rediscovering the Essentiality of Marketing. Developments in Marketing Science: Proceedings of the Academy of Marketing Science. Springer, Cham. Dol: https://doi.org/10.1007/978-3319-29877-1 167.

* Membrado Martínez, J. (2002). Innovación y mejora continua según el modelo EFQM DE EXCELENCIA. Madrid, España: Ediciones Díaz de Santos.

* NOVIS México. (2012). RCD Resorts: Lujo y calidad hotelera que prefieren la dupla SAP y NOVIS. Recuperado de: http://www.novis.mx/mx/mexico/casos-de-exito/rcdresorts-lujo-y-calidad-hotelera-que-prefieren-la-dupla-sap-y-novis/.

* Orantes, S. D., Aguillón, E. y Vázquez, G. (2015). Cómputo en la Nube una opción para PYMES en México. Décima Cuarta Conferencia Iberoamericana en Sistemas, Cibernética e Informática (CISCI).

* Rico Marsard, L. F. y Alucema, M. A. (2010). Conocimiento previo de lugares patrimoniales de México y valores atribuidos por estudiantes de Administración hotelera. El Periplo Sustentable, 19, Julio/Diciembre 2010, 123-146. Recuperado de: https://rperiplo.uaemex.mx/article/download/5028/3618/. 
Schegg, R., Scaglione, M., Liebrich, A. y Murphy, J. (2007). Internet Use by Hospitality SMES in Alpine Destinations. En Sigala M., Mich L., Murphy J. (Eds.), Information and Communication Technologies in Tourism 2007 (pp. 469-480). Vienna: Springer.

* Secretaría de Turismo en México (SECTUR). (2014). Recuperado de: https://www. gob.mx/secturl.

* Stauss, B., Gouthier M. y Seide, W. (2007). Satisfaction Measurement within the Customer Relationship Life Cycle. Chapter Advances in Services Innovations (pp. 205-220). Berlin-Heidelberg: Springer.

Sunset World. (2015). Recuperado de: www.sunsetworld.net.

* Universidad de Rosario (s.f) ¿Qué es el mercadeo relacional? Universidad, ciencia y desarrollo, programa de divulgación científica. Recuperado de http://www. urosario.edu.co/Universidad-Ciencia-Desarrollo/ur/Fasciculos-Anteriores/Tomo$\underline{\|-2007 / F a s c i c u l o-12 / \text { r/Que-es-el-mercadeo-relacional/ }}$

- Zineldin, M. (2000). Beyond Relationship Marketing: Technological ship Marketing. Marketing Intelligence \& Planning, 18(1), 9-23. Dol: https://doi. org/10.1108/02634500010308549.

\section{Cómo citar este artículo}

* Orantes Jiménez, Sandra Dinora, Vázquez Álvarez, Graciela y Tejeida Padilla, Ricardo (2019). Acercar a los hoteles con sus clientes mediante las TIC. Revista Digital Universitaria (RDU). Vol. 20, núm. 3 mayo-junio. DOI: http://doi.org/10.22201/ codeic.16076079e.2019.v20n3.a2. 